\title{
Targeted treatment of acute myeloid leukemia in older adults: role of gemtuzumab ozogamicin
}

\author{
Hien K Duong \\ Mikkael A Sekeres \\ Cleveland Clinic Taussig Cancer \\ Institute Cleveland, Ohio USA
}

Correspondence: Mikkael A Sekeres Department of Hematologic Oncology and Blood Disorders, Cleveland Clinic Taussig Cancer Institute, 9500 Euclid Avenue, Desk R35, Cleveland, Ohio 44I 95 USA

Tel + I 2164459353

Fax +I 2164449464

Email sekerem@ccf.org

\begin{abstract}
As the overall prognosis and treatment response rate to standard chemotherapy for acute myeloid leukemia (AML) remains poor in the older adult population, there is a need for more effective therapeutic agents with lower toxicity profiles that can be offered to these patients. Gemtuzumab ozogamicin (GO) is an anti-CD33 monoclonal antibody that was approved by the US Food and Drug Administration for use as monotherapy in patients 60 years of age and older with relapsed AML. GO consists of a humanized anti-CD33 antibody (hP67.6) which is linked to N-acetyl- $\gamma$ calicheamicin 1,2-dimethyl hydrazine dichloride. Once the antibody attaches to the surface antigen, it is rapidly internalized. Calicheamicin, a potent enediyne, is subsequently released and acts as a cytotoxic anti-tumor agent. In this population, GO has an acceptable toxicity and yields response rates approaching 30\%. The efficacy of GO as monotherapy and in combination therapy for treatment of both de novo and relapsed AML continues to be investigated.
\end{abstract}

Keywords: acute myeloid leukemia therapy, older adults, gemtuzumab ozogamicin

\section{Background - acute myeloid leukemia}

Acute myeloid leukemia (AML) is the most common type of acute leukemia occurring in adults. There will be an estimated 13,290 new diagnoses in the United States in 2008, with a yearly, age-adjusted incidence rate of 3.6 per 100,000 people. ${ }^{1}$ The median age at diagnosis is approximately 67 years, and the incidence is rising, particularly in the "very old" (patients in their 8th and 9th decades of life), likely related to the aging population as a whole. Thus, the majority of AML is seen in older adults.

Initial treatment has not changed significantly over the last 30 years. Standard regimens use cytarabine combined with anthracyclines or anthracenediones for remission induction, followed by cytarabine, alone or in combination, for post-remission therapy. Although there has been little change in chemotherapy regimen, supportive pharmacologic care (including antiemetics, and antibiotics) has improved significantly and has made this chemotherapy more tolerable. The rate of relapse, however, remains high, and the overall outcome in older adult populations is poor. Conventional chemotherapy regimens induce complete remission (CR) in $65 \%$ to $85 \%$ of patients younger than 60 years of age. ${ }^{2-8}$ Of those achieving a CR, only $30 \%$ to $40 \%$ can expect long-term disease-free survival (DFS). In older adults ( $\geq 60$ years of age), results are even more dismal, with CR achievable in $40 \%$ to $55 \%$ of patients. Of those who do achieve a CR, only $10 \%$ to $20 \%$ are still alive 3 years out from diagnosis., ${ }^{4-11}$ This low chance of durable remission comes at a price of high treatment-related mortality ( $20 \%$ or higher compared to less than $10 \%$ in the younger adult population). A number of studies have explored more intensive up-front chemotherapy, addition of cytotoxic drugs, and even extended maintenance therapy, without demonstrable improvement in survival outcomes. ${ }^{12-15}$ Thus, for almost all older AML patients, therapy for relapsed disease will be a consideration. 


\section{Pathobiology of AML in older adults}

Why the high relapse rate in older adults? As advances in molecular biology and genetics continue to develop, AML in the older adult population is increasingly recognized as having distinct biological features and disease characteristics from AML in younger adults.

In the older adult population, AML is more likely to arise from proximal bone marrow stem cell disorders (such as MDS) and with leukemia-specific abnormalities in more than one hematopoeitic cell lineage. Approximately $30 \%$ of older patients with de novo AML actually have evidence of trilineage myelodysplasia. ${ }^{17}$ Having an antecedent hematologic disorder is associated with poor response to chemotherapy, prolonged periods of cytopenia, lower $\mathrm{CR}$ and shorter duration of CR.

In older adults, there tends to be a higher incidence of poor-risk cytogenetic abnormalities, such as abnormalities of chromosomes $5,7,8$, or complex cytogenetics, and multiple comorbidities. They are also less likely to have good-risk cytogenetic findings, such as balanced, core-binding factor abnormalities, including $\mathrm{t}(8 ; 21)$; inv $(16)$ and $\mathrm{t}(16 ; 16)$, involving CBFB-MYH11 chimeric product; and the PML-RARa mutation $\mathrm{t}(15 ; 17) .{ }^{18-22}$ Whether newly identified molecular lesions such as the FMS-related tyrosine kinase 3 gene internal transmembrane duplications (FLT3 ITDs) and mutations of the nucleophosmin gene (NPM1) play a role in the older adult population of AML patients has yet to be determined, though preliminary data indicate these abnormalities may have even greater prognostic potential than the core binding factor abnormalities. Ultimately, however, older age seems to predict for higher rates of relapse and significantly shorter OS. ${ }^{23}$

Secondary AML, which is less responsive to chemotherapy, also is more common in this age group, comprising between $25 \%$ and $50 \%$ of cases, compared to $<10 \%$ in younger adults. Secondary AML is associated with poorer outcomes. ${ }^{2,20,24}$

Finally, AML in the older adult population appears to be intrinsically more difficult to treat, with a higher incidence of resistance to conventional chemotherapy. This is mediated by greater expression of genes that confer drug resistance, such as MDR1, the P-glycoprotein (gp170) chemotherapy efflux pump, present in one study in $71 \%$ of myeloblasts in older adults, compared to only $35 \%$ of blasts in younger AML patients. ${ }^{21}$

\section{Choosing therapy of older adults with AML}

\section{The difficult decision}

Because conventional chemotherapy has such dismal outcomes in the older adult population and the treatment-related mortality and morbidity are so high, the decision of therapy in older adults can be a complicated and difficult process, and as much as possible intensive therapy should be directed to specific molecular abnormalities, or avoided with particularly poor risk characteristics. One study explored the effect of time from diagnosis of AML to treatment initiation on CR and OS in over 1300 AML patients. The results showed that delaying therapy in older patients had no impact on these survival outcomes. ${ }^{25}$ Thus, older patients may benefit from waiting for the results of additional testing to return, allowing enrollment into studies that account for cytogenetic findings or that target molecular therapies.

Although indirect data support the use of intensive chemotherapy in older patients (60 years or older), most will derive little benefit from this approach. Only one randomized study, reported two decades ago, has ever shown a survival advantage of remission induction therapy (with daunorubicin, vincristine, and cytarabine) as compared to best supportive care. This trial noted a significant median survival benefit of only 10 weeks for patients receiving remission induction therapy ${ }^{26} \mathrm{~A}$ more recent case-control study showed a survival advantage for giving intensive chemotherapy compared to best supportive care or low-dose approaches of 197 days vs 53 days (hazard ration [HR] 1.88, $\mathrm{p}=0.01$ ). ${ }^{27}$

Because of high treatment-related mortality and poor outcomes, many older patients choose less aggressive therapy, often at the recommendation of their physicians. In a study by Ferrara, et al questionnaires were sent to 32 hematologic institutions involved in the Gruppo Italiano per le Malattie Ematolgiche dell'Adulto (GIMEMA) group. The purpose of the study was to assess the various factors that influence therapeutic choice in the older population with relapsed AML. The most frequent factor found to influence therapeutic choice was performance status. Other additional factors included age $>70$ years, duration of CR 1 ( $>$ or $<12$ months), concomitant disease requiring specific treatment. It is interesting to note that other prognostic factors such as cytogenetics, serum LDH, previous MDS or white blood cell count were not considered as heavily in this older population. ${ }^{28}$ In older patients who are not fit for aggressive care, Burnett et al have also demonstrated that low-dose ara-C treatment was superior to best supportive care and hydroxyurea in achieving CR. ${ }^{29}$

\section{Therapy of relapsed AML in older adults}

In the younger adult population, in the setting of relapsed disease, the goal is cure. Intensive, cytarabine-based chemotherapy 
salvage regimens with subsequent allogeneic hematopoietic stem cell transplantation remain preferred treatments. These approaches are likely to be too toxic in older adults with relapsed AML, in whom the goals of therapy can no longer be curative. In those older patients who are fit enough and want to continue with treatment, according to National Comprehensive Cancer Network (NCCN) guidelines (for patients who are 60 years of age and older) the recommendations for salvage therapy are enrollment in clinical trials, repetition of initial induction chemotherapy regimen (if the patient has already enjoyed a durable remission from that therapy), or gemtuzumab ozogamicin.

\section{Gemtuzumab ozogamicin}

Gemtuzumab ozogamicin (GO) is an anti-CD33 monoclonal antibody that is conjugated to a cytotoxic agent, calicheamicin. It was approved by the FDA in May 2000 for use as single-agent therapy in elderly patients (patients $\geq 60$ years old) with first-recurrence CD $33^{+} \mathrm{AML}$ who are not candidates for standard therapy. The approved dose is $9 \mathrm{mg} / \mathrm{m}^{2}$ intravenously (given on days 1 and 15).

\section{The CD33 antigen}

The CD33 antigen is a transmembrane cell-surface glycoprotein (of unknown function) that is specific for myeloid cells. It is a member of the sialic acid-binding immunoglobulinlike lectin (siglec) family. It is found on both mature and immature myeloid cells. It is found on erythroid, megakaryocytic, and multipotent progenitors. It is not present on normal pluripotent hematopoietic stems cells. Because it is absent on normal hematopoietic stem cells and has little expression in non-hematopoietic tissue, the $\mathrm{CD}_{3} 3^{+}$ antigen is a reasonable target for monoclonal antibodies against AML.

CD33 is expressed on approximately $90 \%$ of myeloblasts in patients with AML, as defined by the presence of antigen on greater than $20 \%$ leukemic blasts. It is also found on leukemic blasts in a majority of patients with MDS. When $\mathrm{CD}_{3}{ }^{+}$intensity was compared in patients with AML, it was found to be significantly higher in patients $<60$ years of age compared to those who were older. However, levels of $\mathrm{CD}_{3} 3^{+}$intensity did not correlate with cytogenetics, response to therapy, or overall survival. ${ }^{30}$

\section{GO pharmacology}

GO is a humanized IgG4 anti-CD33 monoclonal antibody (hP67.6) conjugated to $\mathrm{N}$-acetyl- $\gamma$ calicheamicin dimethyl hydrazide, a derivative of calicheamicin.
The IgG4 isotype was chosen because it has the longest circulating half-life of all isotypes and is the least likely to participate in immune-mediated mechanisms like complement fixation and antibody-dependent cellular toxicity. The hP67.6 antibody is non-cytotoxic by itself. Calicheamicin is a naturally occurring enediyne antibiotic isolated from actinomycete Micromonospora echinospora calichensis. Enediyne antibiotics are unique reactive compounds; once aromatized, they produce cytotoxic biradicals that cause phophodiester bond DNA breakage. This conjugation allows a favorable balance between hydrolytic stability in physiological buffers ( $\mathrm{pH} 7.4)$ and efficient drug release at $\mathrm{pH}$ of lysosomes $(\mathrm{pH} 4)$.

GO is internalized rapidly after infusion. The endocytosed anti-CD33 complexes translocate to lysosomes where the acidic environment causes release of the calicheamicin derivative. A reactive intermediate of the caclicheamicin is formed through reduction by glutathione which causes double-strand breaks in DNA.

Gender and age do not appear to effect GO pharmacokinetics. There are no significant differences between younger and older patients in terms of maximum concentration, time to maximum concentration, area under the curve, clearance, or volume of distribution of $\mathrm{GO} .^{31}$

\section{GO as single-agent therapy in older patients with relapsed AML}

In a phase 1 study by Sievers et al GO as a single agent was used to treat 40 patients with refractory or relapsed AML. The doses ranged from 0.25 to $9 \mathrm{mg} / \mathrm{m}^{2}$. Patients were included in the study if they had good performance status, a white blood cell count $<30 \times 10^{9} / \mathrm{L}$, and no evidence of renal or liver dysfunction. Of these patients, 18 had undergone prior allogeneic stem cell transplant. Twenty percent achieved a reduction in marrow blast count, with less than 5\% blasts at morphologic exam. Toxicities were mostly infusion-related fevers and chills, and myelosuppression. Approximately $20 \%$ of patients also developed mild elevations of liver transaminases. ${ }^{32}$

The study above then led to 3 open-label multicenter phase II trials, with GO given at a dose of $9 \mathrm{mg} / \mathrm{m}^{2}$ (see Table 1). Because the study designs, endpoints, patient demographics, and dosing schedules were similar, the patients were pooled together to attain a larger efficacy population. From reports in 2001 by Sievers et $\mathrm{al}^{33}$ and by Larson et al in $2005,{ }^{34}$ a total of 277 AML patients were enrolled. These patients were all in first relapse and had evidence of $\mathrm{CD} 33^{+} \mathrm{AML}$ blasts. The median age was 61 years old. The primary end point was the number of patients attaining $\mathrm{CR}$, and secondary end 
Table I Phase II studies of GO monotherapy in relapsed and refractory acute myeloid leukemia

\begin{tabular}{|c|c|c|c|c|c|c|}
\hline Ref. & $\begin{array}{l}\text { Number } \\
\text { of patients }\end{array}$ & $\begin{array}{l}\text { Median age } \\
\text { (range) }\end{array}$ & GO dose & CR/CRp & $\begin{array}{l}\text { Median OS } \\
\text { (months) }\end{array}$ & RFS (months) \\
\hline Larson et $\mathrm{al}^{34}$ & 277 & 61 (20-87) & $\begin{array}{l}9 \mathrm{mg} / \mathrm{m}^{2} \text { on days } \\
\mathrm{I} \text { and } \mathrm{I} 4\end{array}$ & $13 / 13$ & $\begin{array}{l}4.9 \text { (all patients) } \\
\text { I2.2 (CR patients) } \\
\text { I2.9 (CRp patients) }\end{array}$ & $\begin{array}{l}5.2 \text { (all patients) } \\
6.4 \text { (CR patients) } \\
4.5 \text { (CRp patients) }\end{array}$ \\
\hline Taksin et $\mathrm{a}^{56}$ & 57 & $64(22-80)$ & $\begin{array}{l}3 \mathrm{mg} / \mathrm{m}^{2} \text { on days } \\
\mathrm{I}, 4 \text {, and } 7\end{array}$ & $26 / 7$ & 8.4 (all patients) & II (all patients) \\
\hline Piccaluga et $\mathrm{al}^{53}$ & 24 & $63(20-75)$ & $\begin{array}{l}\text { Either } 9 \mathrm{mg} / \mathrm{m}^{2} \text { or } \\
6 \mathrm{mg} / \mathrm{m}^{2} \text { for } 3 \text { days, } \\
\text { at least } 14 \text { days apart }\end{array}$ & $3 / 2$ & 2 (all patients) & - \\
\hline
\end{tabular}

Abbreviations: GO, gemtuzumab ozogamicin; CR, complete remission; CRp, complete remission with incomplete platelet recovery; OS, overall survival; RFS, relapse-free survival.

points were the rates of complete remission with persistent thrombocytopenia (CRp), relapse-free survival (RFS), overall survival (OS), and time to platelet- and time to ANC recovery. The overall response rate was $26 \%(13 \%$ achieved CR and $13 \%$ achieved $\mathrm{CRp}$ ), with a median time to remission of 60 days. Interestingly, there were no differences in response rates among patients stratified by age or cytogenetic abnormalities. The median RFS was 6.4 months for patients who achieved CR and 4.5 months for patients who achieved a CRp. Toxicities included infusion-related fevers and chills, myelosuppression, and about $30 \%$ of patients developed treatment-related infections and/or elevations of bilirubin and transaminases.

In a meta-analysis by Leopold et al, GO as monotherapy was compared to high-dose cytosine arabinoside (HiDAC) in patients with first-relapse AML. The outcome of 128 patients historically treated with HiDAC-containing chemotherapy regimens were compared to 128 patients (enrolled in multiple phase II trials) who were treated with GO $\left(9 \mathrm{mg} / \mathrm{m}^{2}\right.$ on days 1 and 15). The median age of patients receiving GO was significantly higher (60 years of age) than those treated with HiDAC (50 years of age). Of those receiving GO, 41\% of patients achieved a CR or CRp. If the first remission was between 10 and 19 months, there were no differences between the two groups for remission duration following treatment. However, if the first remission duration was 3 to 10 months, then patients treated with GO had higher remission duration following treatment. Interestingly, there was no statistical difference between overall remission rates with $\mathrm{GO}$ or HiDAC containing chemotherapy regimens between the different cytogenetic groups. Interestingly, the analysis revealed that early death rate occurred with HiDAC if age was less than 45 years, whereas lower early death rate resulted from GO therapy in patients who were greater than 75 years of age. ${ }^{35}$ It is important to note that this was not a randomized study and the median ages of these two groups were significantly different, likely reflecting that few older patients are considered candidates for HiDAC.

\section{Toxicity}

GO is generally tolerated well. The most common infusionrelated reactions include chills, fevers, and hypotension that can occur acutely. In the pivotal phase II studies which led to FDA-approval of GO, Larson et al analyzed the efficacy and safety of GO and found that the incidence of Grade 3 or 4 infusion-related events included fevers (6\%), chills (8\%), hypotension ( $4 \%$ ), nausea (3\%), and hypertension $(2 \%){ }^{34}$ The hypotension occurred several hours after completion of infusion and was reversible with intravenous fluid administration. The incidence of infusion-related events was lower on repeat administration. Preventive therapy with corticosteroids and acetaminophen and diphenhydramine were effective in reducing infusion related toxicities. There was no difference in incidence of infusion-related reactions between younger and older patients.

Myelosuppression was seen in the majority of patients. In phase II trials, grade 3 or 4 neutropenia was observed in $98 \%$ of patients. However, the incidence of grade 3 or 4 sepsis (17\%) and pneumonia ( $8 \%$ ) was lower than would be expected. There was no significant difference in the incidence of infections or in the median time to absolute neutrophil recovery (to $500 / \mu \mathrm{L}$ ) between younger and older patients. Despite the observation of grade 3 or 4 thrombocytopenia in $99 \%$ of patients, the overall incidence of grade 3 or 4 bleeding events was only $13 \%$. In younger and older patients who achieved CR, platelet count to $25,000 / \mu \mathrm{L}$ occurred in a median of 35 days and 38 days, respectively. In younger and older patients who achieved a CRp, platelet count to $25,000 / \mu \mathrm{L}$ occurred in 39 days and 72 days, respectively. ${ }^{34}$ 
Elevations in hepatic transaminases and bilirubin were also observed. These were mostly transient events. Grade 3 or 4 hyperbilirubinemia occurred in $29 \%$ of patients, elevations in hepatic aspartate aminotransferase occurred in $18 \%$, and elevations in alanine amino-trasnsferase in $9 \%$. Veno-occlusive disease (VOD) occurred in $16(5 \%)$ patients, eight patients died of this condition. Patients who undergo subsequent myeloablative HSCT were found to have higher incidence of developing VOD. There was no significant difference in incidence of hepatotoxicity between younger and older patients. ${ }^{34}$

\section{GO as combination therapy in older adults with relapsed $A M L$}

Several trials have evaluated efficacy of GO in combination with cytotoxic chemotherapy as first-line therapy, and for treating refractory/relapsed AML (see Table 2). GO has been studied in combination with cytosine arabinoside (ara-C) alone; idarubicin and ara-C; liposome-encapsulated daunorubicin, ara-C, and cyclosporine; fludarabine, ara-C and cyclosporine; and others. ${ }^{36-40}$ The results have been variable, likely reflecting the heterogeneity of these diseases.

In a phase II study by Tsimberidou et al the efficacy of GO used in combination with fludarabine, Ara-C, and cyclosporine (MFAC) in patients with relapsed or refractory AML was investigated. The median age was 53 years, with 11 of the patients older than 60 years of age. Thirty-two patients were included in this study (34\% with refractory disease, $66 \%$ with relapsed AML). Nine (28\%) patients achieved CR and $2(6 \%)$ achieved CRp. Of those achieving CR or CRp, $4(36 \%)$ patients were 60 years or older. The median OS was 5.3 months. Significant side effects were grade 3 or 4 hyperbilirubinemia (44\%), grade 3 or 4 elevated tramsaminases $(18 \%)$, and VOD $(9 \%) .{ }^{36}$
Stone et al in 2004, in a CALGB study, evaluated the efficacy of treating patients with relapsed or refractory AML with high-dose ara-C (HiDAC) in combination with $\mathrm{GO}$ at different doses $\left(9\right.$ or $\left.4.5 \mathrm{mg} / \mathrm{m}^{2}\right)$. The median age was 64 years. In the initial cohort of patients treated with HiDAC $3.0 \mathrm{~g} / \mathrm{m}^{2}$ (over 3 hours daily for 5 days) and GO $9 \mathrm{mg} / \mathrm{m}^{2}$ (on day 1) and $4.5 \mathrm{mg} / \mathrm{m}^{2}$ (on day 8) significant toxicity was seen; 8 of the 9 patients died during induction therapy. In the phase II study, 44 patients were treated with HiDAC $3.0 \mathrm{~g} / \mathrm{m}^{2}$ (over 3 hours daily for 5 days) and GO at $9 \mathrm{mg} / \mathrm{m}^{2}$ (on day 7). Seven (19\%) achieved CR and two (5\%) achieved CRp. None of these patients developed VOD. ${ }^{37}$

Recently, Doyen et al evaluated the efficacy of GO and cytarabine in older patients with relapsed or refractory AML. Fourteen patients were included in the study and the median age was 70 years. Seven patients had de novo AML and 7 had secondary AML. All patients received GO $\left(6 \mathrm{mg} / \mathrm{m}^{2}\right.$ on day 1 and $4 \mathrm{mg} / \mathrm{m}^{2}$ on day 8$)$ and cytarabine (100 mg/m²/24 hours on days $1-7)$. Two patients achieved $\mathrm{CR}$, one achieved $\mathrm{CRp}$, and one achieved PR with an overall response rate of $28 \%$. The median OS was 4.6 months. Treatment-related death occurred in 4 (28\%) patients. ${ }^{38}$

Fianchi et al evaluated the efficacy of GO in combination with ara-C and G-CSF (G-AraMy regimen) in the treatment of elderly patients with poor-prognosis AML. Fifty-three patients, with either untreated or relapsed/primary refractory AML, were included in this study. The median age was 69 years. The OR rate was $57 \%$. Twenty-three patients $(43 \%)$ achieved CR and 1 patient (2\%) achieved CRp. Eleven patients (21\%) achieved PR and, following consolidation, 10 patients achieved CR. Median DFS was 8 months (range or 2-23+ months) and OS was 9 months (range 2-24 + months). The most common side effect

Table 2 Phase I and II studies of GO combination chemotherapy in relapsed and refractory acute myeloid leukemia

\begin{tabular}{|c|c|c|c|c|c|c|}
\hline Ref. & $\begin{array}{l}\text { Number } \\
\text { of patients }\end{array}$ & $\begin{array}{l}\text { Median age } \\
\text { (range) }\end{array}$ & $\begin{array}{l}\text { Other } \\
\text { chemotherapy }\end{array}$ & GO dose & CR/CRp & $\begin{array}{l}\text { Median OS } \\
\text { (months) }\end{array}$ \\
\hline Tsimberidou et $\mathrm{al}^{36}$ & 32 & $53(18-78)$ & $\begin{array}{l}\text { Fludarabine,Ara-C, } \\
\text { cyclosporine }\end{array}$ & $4.5 \mathrm{mg} / \mathrm{m}^{2}$ on day I & $9 / 2$ & 5.3 \\
\hline Chevallier et $\mathrm{a}^{40}$ & 62 & $55.5(|6-7|)$ & Mitoxantrone, Ara-C & $9 \mathrm{mg} / \mathrm{m}^{2}$ on day 4 & $31 / 8$ & 9.5 \\
\hline Stone et a ${ }^{37}$ & 44 & $64(55-69)$ & HiDAC & $9 \mathrm{mg} / \mathrm{m}^{2}$ on day 7 & $7 / 2$ & Not yet reported \\
\hline Cortes et al ${ }^{54}$ & 17 & $55(23-75)$ & $\begin{array}{l}\text { Topotecan, } \\
\text { cytarabine }\end{array}$ & $9 \mathrm{mg} / \mathrm{m}^{2}$ on day I & $2 / 0$ & 8.2 weeks \\
\hline Alvarado et al ${ }^{55}$ & 14 & $6 \mathrm{I}(34-74)$ & $\begin{array}{l}\text { Idarabucin, } \\
\text { cytarabine }\end{array}$ & $\begin{array}{l}6 \mathrm{mg} / \mathrm{m}^{2} \text { on days I } \\
\text { and } \mathrm{I} 5\end{array}$ & $3 / 3$ & 2 \\
\hline Fianchi et $\mathrm{a}^{39}$ & 53 & $69(65-77)$ & Cytarabine, G-CSF & $6 \mathrm{mg} / \mathrm{m}^{2}$ on day 9 & $23 / 1$ & 9 \\
\hline
\end{tabular}

Abbreviations: GO, gemtuzumab ozogamicin; CR, complete remission; CRp, complete remission with incomplete platelet recovery; OS, overall survival; RFS, relapse-free survival. 
was myelosuppression. This study is also interesting in that these responses were obtained without the use of anthracyclines, which can be especially toxic to older patients. Seven patients (13\%) died during induction. The authors concluded that G-AraMy could be a useful treatment approach, with acceptable toxicity, for poor-risk elderly AML patients. ${ }^{39}$

In a phase II study by Chevallier et al GO was combined with intermediate doses of ara-C and mitoxantrone (MIDAM regimen). Sixty-two patients were included in the study (18 patients with refractory disease and 44 patients with relapsed disease). The median age was 55.5 years, 22 patients were 60 years of age or older. The MIDAM regimen consisted of GO $\left(9 \mathrm{mg} / \mathrm{m}^{2}\right)$ on day 4 , cytarabine $\left(1 \mathrm{~g} / \mathrm{m}^{2}\right)$ every 12 hours on days 1 through 5 , and mitoxantrone $\left(12 \mathrm{mg} / \mathrm{m}^{2} /\right.$ day $)$ on days 1 through 3 . The median duration of follow-up was 26.5 months. The overall response rate was 63\%; 31 (50\%) patients achieved CR and $8(13 \%)$ patients achieved CRp. Of those achieving CR or CRp, 15 (24\%) were 60 years of age or older. Grade 3 to 4 hyperbilirubinemia occurred in $16 \%$ of patients and $3 \%$ developed VOD. ${ }^{40}$

\section{GO as front-line therapy in older adults with AML}

There are several studies that have evaluated the efficacy of GO in untreated AML. A phase II trial evaluating the efficacy of GO as monotherapy for induction, consolidation, and maintenance therapy was done by Nabhan et al in 2005. Twelve patients were included in the study. All were over the age of 65 years and none had received any prior treatment for AML. Standard doses of GO $\left(9 \mathrm{mg} / \mathrm{m}^{2}\right)$ were used as induction therapy. For consolidation, GO $\left(6 \mathrm{mg} / \mathrm{m}^{2}\right)$ was given 45 to 60 days after chemotherapy and following recovery of peripheral counts. For maintenance, $\mathrm{GO}\left(3 \mathrm{mg} / \mathrm{m}^{2}\right)$ was given every 4 weeks for 4 cycles. Three (27\%) patients achieved CR. The toxicity profile was acceptable, with myelosuppression being the most common adverse effect. Five of the patients had transient elevation of transaminases, but none had grade 3 or 4 hyperbilirubinemia. None of the patients developed VOD. ${ }^{41}$

Another study by GIMEMA-European Organization for Research and Treatment of Cancer (EORTC) groups investigated the use of GO as single-agent therapy in induction therapy in elderly patients (AML-15B). Patients were eligible for the study if they were $>75$ years of age and had a World Health Organization performance score (PS) of 0-2, or an age between 61 and 75 years with a WHO PS $>2$. Over $50 \%$ of these patients were over the age of 50 years and were deemed to be too frail for other therapy. Forty patients received GO $\left(9 \mathrm{mg} / \mathrm{m}^{2}\right)$ in two doses given 2 weeks apart. Seven patients achieved either a CR or CRp (17\%), 24 patients had resistant disease (60\%), and 7 patients died during induction (17\%). The median OS was 4.3 months; 1-year survival was 34\%; and 2 -year survival was 7\%. Toxicities included myelosuppression (a majority of patients), and severe liver toxicity (4 patients, only 1 died of fatal VOD). From this study, it was concluded that GO given at this dose was too toxic for patients over the age of 75 years and dose-reduction should be strongly considered. ${ }^{42}$

Estey et al evaluated GO with or without interleukin 11 (IL-11) compared to patients who had received idarubicin and continuous-infusion, high-dose cytarabine (IA). Fifty-one patients were included in the study and the median age was 72 years. Patients were randomized to received induction therapy with GO $(9 \mathrm{mg} / \mathrm{m} 2$ on days 1 and 8$)$ with or without IL-11 $(15 \mu \mathrm{g} / \mathrm{kg}$ on days 3-28). Two of 26 patients who received $\mathrm{GO}$ achieved $\mathrm{CR}$. Nine of 25 patients who received GO and IL-11 achieved CR. This was compared to patients who had received IA. Patients who had received IA demonstrated higher CR rate and survival rates. ${ }^{43}$

Clavio, et al evaluated the efficacy of adding low-dose GO to fludarabine, ara-C, and idarubicin (MY-FLAI regimen) in patients with newly diagnosed CD33 ${ }^{+}$AML. This was a prospective, multi-center trial in comparison with a historical cohort of patients who had received FLAI, without GO. Forty-six patients were treated with MY-FLAI and compared with a historical cohort of 47 patients (matched for age and cytogenetics) who received FLAI. The median age was 66 years. There was no significant difference in CR rate between the groups. However, patients with de novo AML receiving GO had lower risk of relapse at 2 years ( $40 \%$ vs $80 \%, \mathrm{p}=0.01$ ) and also demonstrated significantly better overall survival ( $40 \%$ vs $14 \%, p=0.02)$. A higher incidence of induction-related deaths were actually reported in the historical cohort of patients only receiving GO than compared to those who received the MY-FLAI regimen. This data suggested that adding GO might prolong DFS, particularly in patients with de novo AML, with acceptable toxicity. ${ }^{44}$

In another phase II study, Nand et al reported on the use of a combination of hydroxyurea, azacitidine, and low-dose GO in elderly patients with untreated non-M3 AML and high-risk MDS. Twenty patients were enrolled in the study (17 patients had AML and 3 had MDS RAEB II). The median age was 76 years. The hydroxyurea was administered in order to reduce the white blood cell count to below $10,000 / \mu \mathrm{L}$ and allow for a lower GO dose $\left(3 \mathrm{mg} / \mathrm{m}^{2}\right)$, in attempts to decrease toxicity. Eleven patients (55\%) achieved a CR and 3 achieved 
CRp (15\%). The median DFS was 8 months and median OS was 10 months. Only 1 patient died early on due to disease progression. A majority of these patients were able to be treated in the outpatient setting. ${ }^{45}$

\section{GO in older adults with APL}

In patients with acute promyelocytic leukemia (APL), the addition of GO can produce molecular remission. APL blasts typically have high expressions of CD33 antigen and they also have low levels of P-glycoprotein (Pgp), which is a product of the MDR1 gene which is a known mediator of multidrug resistance. GO has been found to be highly effective in treating patients with APL in relapse, and is an active area of research to avoid the use of anthracyclines and cytarabine, a particularly appealing approach for the rare older adult with APL.

Lo-Coco et al evaluated GO as monotherapy for molecularly relapsed APL. Among 16 patients included in the study, 7 were older than 65 years of age. All patients had received prior therapy of ATRA and anthracycline-based therapy with cytarabine. All patients had demonstrated molecular relapse. These patients were treated with $\mathrm{GO}$ at $6 \mathrm{mg} / \mathrm{m}^{2}$ on days 1 and 15 . Fourteen (88\%) patients responded and 7 (44\%) of these patients remained in sustained molecular remission for median of 15 months. The estimated OS after first GO treatment was $74 \%$ and molecular RFS was $43 \%$. These results supported that GO could be used as monotherapy in patients with molecular relapse of APL. ${ }^{46}$

Estey et al evaluated GO and all-trans retinoic acid (ATRA) in patients with previously untreated APL. GO was administered at a dose of $9 \mathrm{mg} / \mathrm{m}^{2}$ on day 1 or day 5 of therapy. Nineteen patients were included in the study. Three patients also received idarubicin due to elevated white blood count $>30,000$. Once CR was achieved, patients received 8 courses of GO ( $9 \mathrm{mg} / \mathrm{m}^{2}$ every $4-5$ weeks $)$ and ATRA. If there was persistent or recurrent polymerase chain reaction (PCR) positivity, idarubicin was added to the maintenance regimen. Sixteen of the nineteen $(84 \%)$ patients achieved CR. Fourteen of the 16 (88\%) patients given GO and ATRA without idarubicin achieved CR. GO was well tolerated. These data suggested that GO could be effective therapy in patients with untreated APL. ${ }^{47}$

Estey et al also investigated the efficacy of GO combined with ATRA versus arsenic trioxide (ATO) and ATRA in patients with previously untreated APL. Among 44 patients with a median age of 49 years, 25 low-risk patients received ATO and ATRA for induction and, after achieving CR, received ATO plus ATRA without chemotherapy, while
19 high-risk patients received the identical treatment, but also received GO $\left(9 \mathrm{mg} / \mathrm{m}^{2}\right)$ on day 1 of induction. Thirty-nine of the 44 patients achieved CR (24 of 25 in the low-risk, 15 of 19 in the high-risk). These results suggest that ATRA plus ATO may be an effective alternative to chemotherapy in patients with low-risk, previously untreated APL. It also suggests that ATRA plus ATO in combination with GO may be beneficial in patients with high-risk, previously untreated APL. ${ }^{48}$

Aribi et al investigated efficacy of GO in combination with ATO and ATRA in patients with APL in first relapse. Eight patients were enrolled in the study. Two (25\%) patients were greater than 60 years. All patients had received previous treatment with ATRA as single-agent or in combination with other chemotherapy. Seven of these patients had hematologic relapse, and one had a molecular relapse. All patients achieved molecular CR. After a median follow-up of $\geq 36$ months, 6 patients remained alive in CR and 2 patients died in CR. Of the 2 patients who died, 1 died due to complications of metastatic lung adenocarcinoma, and the other of sepsis. ${ }^{49}$

Interestingly, in these patients with APL, the side effects were minimal and no cases of VOD have been reported. ${ }^{49}$

\section{Resistance to GO}

Sensitivity to GO and mechanisms of resistance to GO continue to be investigated. Initial in vitro investigations with GO were performed on 4 different human AML lines (KG1, THP-1, HL-60, and NB4) and subsequently in primary samples from patients with AML. GO was found to produce three different responses in AML cell lines: arrest in G2 phase of cell cycle followed by apoptosis, G2 arrest with little apoptosis, and resistance to high doses of GO. These different responses did not correlate with CD33 expression or multiple drug resistance (MDR) protein expression..$^{50}$

Recently, CD33 expression and P-glycoprotein (P-gp)-mediated drug efflux have been shown to inversely correlate with response to GO. In this particular study by Water et al, CD33 expression and P-gp activity were found to be inversely correlated. Also, patients with lower P-gp activity demonstrated greater response to $\mathrm{GO}^{51}$ However, it is possible that the effect against GO and calicheamicin occurs through other antiapoptotic mechanisms as well. ${ }^{52}$

\section{Other approaches to relapsed AML in older adults}

There are several other therapy approaches currently being investigated in older adults. Clofarabine, a purine nucleoside analog, is being studied as front-line therapy in older patients 
with AML. Decitabine and azacitidine are hypomethylating agents also being studied in de novo and relapsed AML. FLT3 ITD mutations, as mentioned before, have been found to be present in up to one-third of AML cell lines. These mutations result in unregulated cell proliferation. There are several oral FLT3 inhibitors that are currently under clinical investigation in relapsed/refractory AML and front-line in older patients who are considered "unfit" for intensive chemotherapy. Tipifarnib, an oral farnesyltransferase inhibitor (FTI), has also been studied in older patients with refractory or relapsed AML. FTIs are selective inhibitors of intracellular farnesyltransferase, which is an enzyme that catalyzes transfer of farnesyl to cysteine terminal residue of various substrate proteins (including oncoprotein Ras and several others). These inhibitors subsequently inhibit cellular signaling transduction pathways and can potentially inhibit tumor growth.

\section{Conclusions}

GO is a humanized anti-CD33 monoclonal antibody therapy that has been shown to be affective and well tolerated in the older adult population. It has acceptable toxicity profile; the main adverse effects include infusion-related events, severe myelosuppression, and hepatotoxicity (including VOD). GO is an important new anti-leukemic therapy and has promising efficacy in both de novo and relapsed disease. It will be important to better define the population of patients that would benefit most from this targeted therapy. Also, the role of GO in monotherapy or in combination therapy regimens for the treatment of AML needs to be further investigated.

\section{Disclosures}

The authors disclose no conflicts of interest.

\section{References}

1. National Cancer Institute. SEER (Surveillance, Epidemiology, and End Results) Cancer Statistics Review 1975-2002 [on-line]. Available from URL: http://seer.cancer.gov

2. Baer MR, George SL, Dodge RK, et al. Phase 3 study of the multidrug resistance modulator PSC-833 in previously untreated patients 60 years of age and older with acute myeloid leukemia: Cancer and Leukemia Group B Study 9720. Blood. 2002;100(4):1224-1232.

3. Godwin JE, Kopecky KJ, Head DR, et al. A double-blind placebocontrolled trial of granulocyte colony-stimulating factor in elderly patients with previously untreated acute myeloid leukemia: a Southwest oncology group study (9031). Blood. 1998;91(10):3607-3615.

4. Lowenberg B, Suciu S, Archimbaud E, et al. Mitoxantrone versus daunorubicin in induction-consolidation chemotherapy-the value of low-dose cytarabine for maintenance of remission, and an assessment of prognostic factors in acute myeloid leukemia in the elderly: final report. European Organization for the Research and Treatment of Cancer and the Dutch-Belgian Hemato-Oncology Cooperative Hovon Group. J Clin Oncol. 1998;16(3):872-881.
5. Mayer RJ, Davis RB, Schiffer CA, et al. Intensive postremission chemotherapy in adults with acute myeloid leukemia. Cancer and Leukemia Group B. N Engl J Med. 1994;331(14):896-903.

6. Rowe JM, Neuberg D, Friedenberg W, et al. A phase 3 study of three induction regimens and of priming with GM-CSF in older adults with acute myeloid leukemia: a trial by the Eastern Cooperative Oncology Group. Blood. 2004;103(2):479-485.

7. Stone RM, Berg DT, George SL, et al. Granulocyte-macrophage colonystimulating factor after initial chemotherapy for elderly patients with primary acute myelogenous leukemia. Cancer and Leukemia Group B. N Engl J Med. 1995;332(25):1671-1677.

8. Byrd JC, Mrozek K, Dodge RK, et al. Pretreatment cytogenetic abnormalities are predictive of induction success, cumulative incidence of relapse, and overall survival in adult patients with de novo acute myeloid leukemia: results from Cancer and Leukemia Group B (CALGB 8461). Blood. 2002;100:4325-4336.

9. Lowenberg B, van Putten W, Theobald M, et al. Effect of priming with granulocyte colony-stimulating factor on the outcome of chemotherapy for acute myeloid leukemia. N Engl J Med. 2003;349(8):743-752.

10. Goldstone AH, Burnett AK, Wheatley K, Smith AG, Hutchinson RM, Clark RE. Attempts to improve treatment outcomes in acute myeloid leukemia (AML) in older patients: the results of the United Kingdom Medical Research Council AML11 trial. Blood. 2001;98(5): 1302-1311.

11. Stone RM, Berg DT, George SL, et al. Postremission therapy in older patients with de novo acute myeloid leukemia: a randomized trial comparing mitoxantrone and intermediate-dose cytarabine with standard-dose cytarabine. Blood. 2001;98(3):548-553.

12. Rowe JM, Neuberg D, Friedenberg W, et al. A phase 3 study of three induction regimens and of priming with GM-CSF in older adults with AML: A trial by the Eastern Cooperative Oncology Group. Blood. 2004;103:479-485.

13. Goldstone AH, Burnett AK, Wheatley K, et al. Attempts to improve treatment outcomes in AML in older patients: The results of the United Kingdom Medical Research Council AML11 trial. Blood. 2001;98:1302-1311.

14. Anderson JE, Kopecky KJ, Willman CL, et al. Outcome after induction chemotherapy for older patients with AML is not improved with mitoxantrone and etoposide compared to daunorubicin and cytarabine: A Southwest Oncology Group Study. Blood. 2002;100:3869-3876.

15. Van der Holt B, Lowenberg B, Burnett AK, et al. The value of the MDR1 reversal agent PSC-833 in addition to daunorubicin and cytarabine in the treatment of elderly patients with previously untreated AML in relation to MDR1 status at diagnosis. Blood. 2005;106:2646-2654.

16. Schlenk RF, Dohner K, Krauter J, et al. Mutations and treatment outcome in cytogenetically normal acute myeloid leukemia. $N$ Engl J Med. 2008;358:1909-1918.

17. United States Census Bureau. US Interim Projections by Age, Sex, Race, and Hispanic Origin. http://wwwcensusgov/ipc/www/usinterimproj. 2004. Internet release date March 18, 2004.

18. Bloomfield CD, Lawrence D, Byrd JC, et al. Frequency of prolonged remission duration after high-dose cytarabine intensification in acute myeloid leukemia varies by cytogenetic subtype. Cancer Res. 1998;58(18): 4173-4179.

19. Grimwade D, Walker H, Oliver F, et al. The importance of diagnostic cytogenetics on outcome in AML: analysis of 1,612 patients entered into the MRC AML 10 trial. The Medical Research Council Adult and Children's Leukaemia Working Parties. Blood. 1998;92(7):2322-2333.

20. Grimwade D, Walker H, Harrison G, et al. The predictive value of hierarchical cytogenetic classification in older adults with acute myeloid leukemia (AML): analysis of 1065 patients entered into the United Kingdom Medical Research Council AML11 trial. Blood. 2001;98(5):1312-1320.

21. Leith CP, Kopecky KJ, Godwin J, et al. Acute myeloid leukemia in the elderly: assessment of multidrug resistance (MDR1) and cytogenetics distinguishes biologic subgroups with remarkably distinct responses to standard chemotherapy. A Southwest Oncology Group study. Blood. 1997;89(9):3323-3329. 
22. Tilly $\mathrm{H}$, Castaigne $\mathrm{S}$, Bordessoule $\mathrm{D}$, et al. Low-dose cytarabine versus intensive chemotherapy in the treatment of acute nonlymphocytic leukemia in the elderly. J Clin Oncol. 1990;8(2):272-279.

23. Buchner T, Berdel WE, Haferlach $\mathrm{C}$, et al. Older age is an independent risk factor in AML. ASH Annual Meeting Abstracts. 2008;112:555

24. Farag S, Archer K, Mrózek K, et al. Pre-treatment cytogenetics predict complete remission and long-term outcome in patients (Pts) 60 years with acute myeloid leukemia (AML): results from Cancer and Leukemia Group B (CALGB) 8461. Blood. 2004;104:568 [abstract].

25. Sekeres MA, Elson $P$, Wang $X$, et al. Time from diagnosis to treatment initiation predicts survival in younger, but not older, acute myeloid leukemia patients. Blood. 2009;113:28-36.

26. Lowenberg B, Zittoun R, Kerkhofs H, et al. On the value of remissioninduction chemotherapy in elderly patients of $65+$ years with acute myeloid leukemia: a randomized phase III study of the European Organization for Research and Treatment of Cancer Leukemia Group. J Clin Oncol. 1989;7:1268-1274.

27. Baz R, Rodriguez C, Fu AZ, et al. Impact of remission induction chemotherapy on survival in older adults with acute myeloid leukemia. Cancer. 2007;110:1752-1759.

28. Ferrara F, Annunziat M, Copia C, Magrin S, Mele G, Mirto S Therapeutic options and treatment results for patients over 75 years of age with acute myeloid leukemia. Haematologica. 1998;83: 126-131.

29. Burnett AK, Milligan D, Prentice AG, et al. A comparison of low-dose cytarabine and hydroxyurea with or without all-trans retinoic acid for acute myeloid leukemia and high-risk myelodysplastic syndrome in patients not considered fit for intensive treatment, Cancer. 2007;109(6):1114-1124.

30. Jilani I, Estey E, Huh Y, et al. Differences in CD33 intensity between various myeloid neoplasms. Am J Clin Pathol. 2002;118:560-566.

31. Bradley JM, Dowell JA, King SP, Liu H, Berger MS; Mylotarg Study Group. Impact of age and gender on the pharmacokinetics of gemtuzumab ozogamicin. Pharmacotherapy. 2001;21:1175-1180.

32. Sievers EL, Appelbaum FR, Spielberger RT, et al. Selective ablation of acute myeloid leukemia using antibody-targeted chemotherapy: a phase I study of anti-CD33 calicheamicin immunoconjugate. Blood. 1999;93:3678-3684.

33. Sievers EL, Larson RA, Stadtmauer EA, et al. Mylotarg Study Group. Efficacy and safety of gentuzumab ozogamicin in patients with CD33-positive acute myeloid leukemia in first relapse. J Clin Oncol. 2001;19:3244-3254

34. Larson RA, Sievers EL, Stadtmauer EA, et al. Final report of efficacy and safety of gemtuzumab ozogamicin in patients with CD33-positive acute myeloid leukemia in first recurrence. Cancer. 2005;104: 1442-1452.

35. Leopold LH, Berger MS, Cheng SC, Cortes J, Giles F, Estey E. Comparative efficacy and safety of gemtuzumab ozogamicin monotherapy and high-dose cytarabine combination therapy in the treatment of patients with acute myeloid leukemia in first relapse. Clin $A d v$ Hematol Oncol. 2003;1:112-118.

36. Tsimberidou A, Cortes J, Thomas D, et al. Gemtuzumab ozogamicin, fludarabine, cytarabine and cyclosporine combination regimen in patients with $\mathrm{CD} 33^{+}$primary resistant or relapsed acute myeloid leukemia. Leuk Res. 2003;27:893-897.

37. Stone RM, Moser B, Schulman P, et al. A dose escalation and phase II study of Gemtuzumab Ozogamicin (GO) with high-dose cytarabine (HiDAC) for patients with refractory or relapsed acute myeloid leukemia; CALGB 19902. Blood. 2004;104:Abstr 873.

38. Doyen J, Italiano A, Peyrade F, Bouyer C, Thyss A. Gemtuzumab ozogamicin plus cytarabine in elderly patients with relapsed or refractory acute myeloid leukemia. Brit J Haem 2008;141;734-747.

39. Fianchi L, Pagano L, Leoni F, et al. Gemtuzumab, cytosine arabinoside, G-CSF combination (G-AraMy) in the treatment of elderly patients with poor-prognosis acute myeloid leukemia. Ann Oncol. 2008;19: 128-134.
40. Chevallier P, Delaunay J, Turlure P, et al. Long-term disease-free survival after gemtuzumab, intermediate-dose cytarabine, and mitoxantrone in patients with $\mathrm{CD} 33^{+}$primary resistant or relapsed acute myeloid leukemia. J Clin Oncol. 2008;26(32):5192-5197.

41. Nabhan C, Rundhaugen L, Riley M, et al. Phase II pilot trial of gemtuzumab ozogamicin (GO) as first line therapy in acute myeloid leukemia patients age 65 or older. Leuk Res. 2004;28:909-919.

42. Amadori S, Suciu S, Stasi R, et al. Gemtuzumab ozogamicin (Mylotarg) as single-agent treatment for frail patients 61 years of age and older with acute myeloid leukemia: final results of AML-15B, a phase 2 study of the European Organization for Research and Treatment of Cancer and Gruppo Italiano Malattie Emattologiche dell'Adulto Leukemia Groups. Leukemia. 2005;19:1768-1773.

43. Estey E, Thall PF, Giles FJ, et al. Gemtuzumab ozogamicin with or without interleukin 11 in patients 65 years of age or older with untreated acute myeloid leukemia and high-risk myelodysplastic syndrome: comparison with idarubicin plus continuous-infusion, high-dose cytosine arabinoside. Blood. 2002;99:4343-4349.

44. Clavio M, Vignolo L, Albarello A, et al. Adding low-dose gemtuzumab ozogamicin to fludarabine, Ara-C and idarubicin (MY-FLAI) may improve disease-free and overall survival in elderly patients with nonM3 acute myeloid leukaemia: results of a prospective, pilot, multi-centre trial and comparison with a historical cohort of patients, Br J Haematol. 2007;138:186-195.

45. Nand S, Godwin J, Smith S, et al. Hydroxyurea, azacitidine and gemtuzumab ozogamicin therapy in patients with previously untreated nonM3 acute myeloid leukemia and high-risk myelodysplastic syndromes in the elderly: results from a pilot trial. Leuk Lymphoma. 2008;49(11): 2141-2147.

46. Lo-Coco F, Cimino G, Breccia M, et al. Gemtuzumab ozogamicin (Mylotarg) as a single agent for molecularly relapsed acute promyelocytic leukemia. Blood. 2004;104:1995-1999.

47. Estey EH, Giles FJ, Beran M, et al. Experience with gemtuzumab ozogamycin (mylotarg) and all-trans retinoic acid in untreated acute promyelocytic leukemia. Blood. 2002;99:4222-4224.

48. Estey E, Garcia-Manero G, Ferrajoli A, et al. Use of all-trans retinoic acid plus arsenic trioxide as an alternative to chemotherapy in untreated acute promyelocytic leukemia. Blood. 2006;107:3469-3473.

49. Aribi A, Kantarjian HM, Estey EH, et al. Combination therapy with arsenic trioxide, all-trans retinoic acid, and gemtuzumab ozogamicin in recurrent acute promyelocytic leukemia. Cancer. 2007;109: $1355-1359$.

50. Amico D, Barbui AM, Erba E, Rambaldi A, Introna M, Golay J. Differential response of human acute myeloid leukemia cells to gemtuzumab ozogamicin (Mylotarg ${ }^{\circledR}$ ) in vitro. Role of Chk1 and Chk2 phosphorylation and caspase 3. Blood. 2003;101(11):4589-4597.

51. Walter RB, Gooley TA, van der Velden VHJ, et al. CD33 expression and P-glycoprotein-mediated drug efflux inversely correlate and predict clinical outcome in patients with acute myeloid leukemia treated with gemtuzumab ozogamicin monotherapy. Blood. 2007;109:4168-4170.

52. van der Kolk DM, de Vries EGE, Muller M, Vellenga E. The role of drug efflux pumps in acute myeloid leukemia. Leuk Lymphoma. 2002;43:685-701.

53. Piccaluga PP, Martinelli G, Rondoni M, et al. Gemtuzumab ozogamicin for relapsed and refractory acute myeloid leukemia and myeloid sarcomas. Leuk Lymphoma. 2004;45(9):1791-1795.

54. Cortes J, Tsimberidou A, Alvarez R, et al. Mylotarg combined with topotecan and cytarabine in patients with refractory acute myelogenous leukemia. Cancer Chemother Pharmacol. 2002;50:497-500.

55. Alvarado Y, Tsimberidou A, Kantarjian H, et al. Pilot study of Mylotarg, idarubicin and cytarabine combination regimen in patients with primary resistant or relapsed acute myeloid leukemia. Cancer Chemother Pharmacol. 2003;51:87-90.

56. Taksin AL, Legrand O, Raffoux E, de Revel T, et al. High efficacy and safety profile of fractionated doses of Mylotarg as induction therapy in patients with relapsed acute myeloblastic leukemia: a prospective study of the alpha group. Leukemia. 2007;21:66-71. 
